\title{
Scaffold attachment factor A (SAF-A) and Ku temporally regulate repair of radiation-induced clustered genome lesions
}

\author{
Muralidhar L. Hegde ${ }^{1,2,3, *}$, Arijit Dutta ${ }^{1,4, *}$, Chunying Yang ${ }^{1, *}$, Anil K. Mantha ${ }^{4,8}$, \\ Pavana M. Hegde ${ }^{1}$, Arvind Pandey ${ }^{1}$, Shiladitya Sengupta ${ }^{1,3}$, Yaping Y ${ }^{5}$, Patrick \\ Calsou ${ }^{6}$, David Chen7, Susan P. Lees-Miller ${ }^{5}$ and Sankar Mitra ${ }^{1,3,4}$ \\ ${ }^{1}$ Department of Radiation Oncology, Houston Methodist Research Institute, Houston, TX, USA \\ ${ }^{2}$ Houston Methodist Neurological Institute, Houston, TX, USA \\ ${ }^{3}$ Weill Medical College of Cornell University, Ithaca, NY, USA \\ ${ }^{4}$ Department of Biochemistry and Molecular Biology, University of Texas Medical Branch, Galveston, TX, USA \\ ${ }^{5}$ Department of Biochemistry \& Molecular Biology, University of Calgary, Calgary, Canada \\ ${ }^{6}$ Institut de Pharmacologie et de Biologie Structurale, CNRS, Université de Toulouse-Université Paul Sabatier, Equipe \\ Labellisée Ligue contre le Cancer, Toulouse, France \\ 7 UT Southwestern Medical Center, Dallas, TX, USA \\ ${ }^{8}$ Center for Animal Sciences, School of Basic and Applied Sciences, Central University of Punjab, Bathinda, Punjab, India \\ * These authors have contributed equally to this work \\ Correspondence to: Sankar Mitra, email: smitra2@houstonmethodist.org
}

Muralidhar L. Hegde, email: mlhegde@houstonmethodist.org

Keywords: SAF-A/hnRNP-U, KU, DNA-PK, BER, DSB repair

Received: May 17, $2016 \quad$ Accepted: May 26, 2016

Published: June 09, 2016

\section{ABSTRACT}

Ionizing radiation (IR) induces highly cytotoxic double-strand breaks (DSBs) and also clustered oxidized bases in mammalian genomes. Base excision repair (BER) of bi-stranded oxidized bases could generate additional DSBs as repair intermediates in the vicinity of direct DSBs, leading to loss of DNA fragments. This could be avoided if DSB repair via DNA-PK-mediated nonhomologous end joining (NHEJ) precedes BER initiated by NEIL1 and other DNA glycosylases (DGs). Here we show that DNAPK subunit $\mathrm{Ku}$ inhibits DGs via direct interaction. The scaffold attachment factor (SAF)-A, (also called hnRNP-U), phosphorylated at Ser59 by DNA-PK early after IR treatment, is linked to transient release of chromatin-bound NEIL1, thus preventing BER. SAF-A is subsequently dephosphorylated. Ku inhibition of DGs in vitro is relieved by unphosphorylated SAF-A, but not by the phosphomimetic Asp59 mutant. We thus propose that SAF-A, in concert with $\mathrm{Ku}$, temporally regulates base damage repair in irradiated cell genome.

\section{INTRODUCTION}

Ionizing radiation (IR) as well as radiomimetic drugs induce clusters of damage in the genome, including most cytotoxic double-strand breaks (DSBs), as well as single-strand breaks (SSBs) with nonligatable termini, bi-stranded clusters of abasic (AP) sites, and oxidized bases $[1,2]$. To restore genomic integrity, DSBs activate signalling cascades that begin with the binding of the Mre11/Rad50/Nbs1 (MRN) complex to the DSBs followed by activation of the protein kinase ATM, which subsequently activates the cell cycle checkpoint and DNA repair pathways [3-5]. DSB repair occurs via homologous recombination (HR) in $\mathrm{S} / \mathrm{G}_{2}$ cells and via nonhomologous end joining (NHEJ) in all cells $[4,6$, 7]. NHEJ, the predominant repair process in all cells, is initiated by the binding of $\mathrm{Ku}(\mathrm{Ku} 70 / \mathrm{Ku} 80$ heterodimer) subunit of DNA-dependent protein kinase (DNA-PK) to the DSB site, which then recruits the catalytic subunit (DNA-PKcs). DNA-PK in addition to phosphorylating 
many downstream targets enables formation of a large NHEJ complex comprising DNA ligase4/XRCC4/XLF that joins the DSBs after their end processing [6]. The base lesions and AP sites are repaired via the base excision/SSB repair (BER/SSBR) pathway and involves formation of SSB intermediates, which like the IR-induced direct SSBs bind PARP-1. PARP-1 in turn recruits DNA glycosylases (DGs), AP-endonuclease 1 (APE1), DNA polymerases, and DNA ligase $3 \alpha(\operatorname{Lig} 3 \alpha) / \mathrm{XRCC} 1[8,9]$. While NHEJ is the major pathway for DSB repair [10], alternative NHEJ (Alt-EJ) involving PARP-1 and the BER machinery may also repair DSBs, including those generated during the repair of bi-stranded oxidized lesion clusters; however its contribution to DSB repair is not very clear [11].

How multiple repair pathways, particularly NHEJ and BER, are coordinated in repairing IR-induced damage clusters in the mammalian genome have not been investigated. The SSB intermediates generated during repair of oxidized bases and AP sites, in the proximity of an unrepaired DSB, could cause additional DNA sequence loss [12, 13]. Furthermore, bi-stranded base lesions/AP sites and SSBs could produce secondary DSBs [14-18]. Hence, we hypothesized that NHEJ precedes BER.

Recent studies have documented involvement of non-canonical proteins, in particular, the family of RNA/ DNA binding proteins, e.g., hnRNPs in the repair of both oxidized lesions and DSBs [19, 20]. While hnRNP-U is primarily known for its role in mRNA processing and transport [21], it was independently identified and named scaffold attachment factor (SAF)-A, on the basis of its strong binding to nuclear scaffold/ matrix with affinity for A-T-rich DNA [22]. This $90 \mathrm{kDa}$ protein is the largest member of the abundant hnRNP family. Interestingly, hnRNP-U/SAF-A was also found to be associated with WT1 (Wilms tumor 1) protein and suggested to be a potential Wilms tumor gene [23]. It interacts with MDM2, an E3 ubiquitin ligase involved in degradation of p53, a central player in DNA damage response. We previously characterized the presence of SAF-A/hnRNP-U in the immunoprecipitates (IPs) of both NEIL1 and NEIL2 and its functional implication in BER [19, 20]. More recent studies have documented that irradiation induces its phosphorylation at Ser59 by DNA-PK, followed by its dephosphorylation, presumably after completion of DSB repair [24, 25]. However, the possible involvement of hnRNP-U/SAF-A in radiation-induced clustered damage repair has not been explored. Polo-like kinase 1 also phosphorylates SAF-A at Ser59 which has been implicated in accurate mitosis [26].

In this report, we demonstrate that $\mathrm{Ku}$ negatively regulates BER by inhibiting base excision/strand-scission activity of all oxidized base-specific DGs at IR-induced clustered damage sites to allow the completion of DSB repair via NHEJ. SAF-A then reverses inhibition to activate BER, which is regulated by its IR-responsive phosphorylation-dephosphorylation events. Our experimental observations are consistent with the temporal regulation of NHEJ and BER at IR-induced damage clusters, which is critical for maintaining genomic fidelity in human cells.

\section{RESULTS}

\section{BER contributes to radioresistance in human cells}

To test the role of BER in the repair of IR-induced clustered genome damage, we analysed radiosensitivity of BER-deficient human cells using clonogenic cell survival assay. siRNA-mediated depletion of NEIL1 or APE1 caused a modest reduction in survival of irradiated HEK293 cells (Figure 1A) as compared to that of IRtreated NHEJ-deficient cells $[4,27,28]$. Moderate radioprotective role of BER enzymes was consistently observed in mouse fibroblasts [29, 30]. Furthermore, varying degrees of radiosensitivity associated with BER and NHEJ protein deficiencies [31] suggest generation of distinct subgroups of IR-induced lesions that independently and additively affect cell survival.

\section{Early NHEJ proteins interact with BER enzymes in irradiated cells}

We next tested for the presence of crosstalk between BER and DSB repair machinery in cells after irradiation. Co-immunoprecipitation (co-IP) analysis with FLAG antibody (Ab) from nuclear extract of HEK293 cells stably expressing FLAG-NEIL1, after degradation of nucleic acids, revealed stable binding of early NHEJ proteins Ku70 and DNA-PKcs to NEIL1, whose levels in the IP significantly increased after irradiation (Figure 1B). The absence of $\mathrm{Ku}$ in the IP of NEIL1 C-terminal domain (CTD) deletion mutant (N311)-FLAG indicated that $\mathrm{Ku}$ interaction requires the CTD [which was previously implicated for most of NEIL1's DNA-independent, binary interaction [32]] (Figure 1C). The presence of $\mathrm{Ku}$ in the NEIL1 IP from ethidium bromide (EtBr)-treated cell extracts further confirmed lack of involvement of nucleic acids in the NEIL1-Ku interaction (data not shown). The IP of NEIL2, a related DG that serves as the primary back up for NEIL1 [33], also contained Ku70 and DNA-PKes (Figure 1C). Similarly, the FLAG(WT)-APE1 IP contained Ku70, along with the other BER proteins PARP1 and Lig $3 \alpha$; none of these was detected in the IP of N-terminal deletion mutant APE1 (NA33) (Figure 1D). Analogous to the CTD of NEIL1, the N-terminal domain of APE1 provides the common interaction interface for the BER proteins [34]. XRCC4, a key NHEJ protein, was not detected in the IP of either NEIL1 or APE1. These interactions were also enhanced after treatment with radiomimetic bleomycin. The FLAG 
A

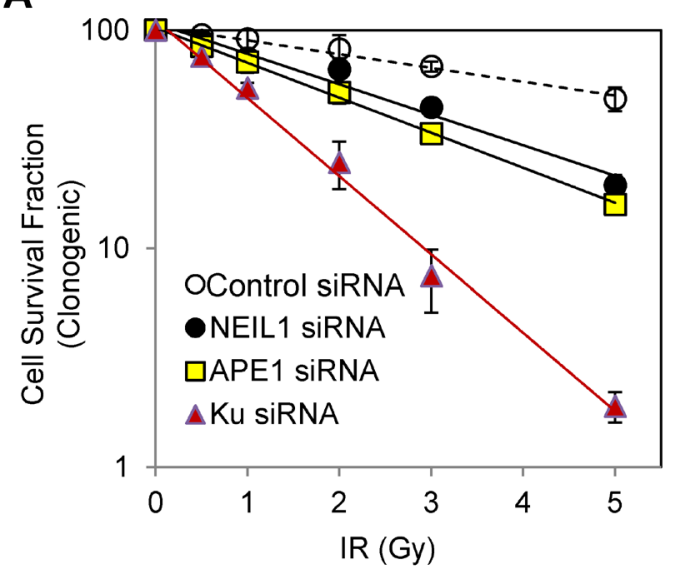

SiRNA B
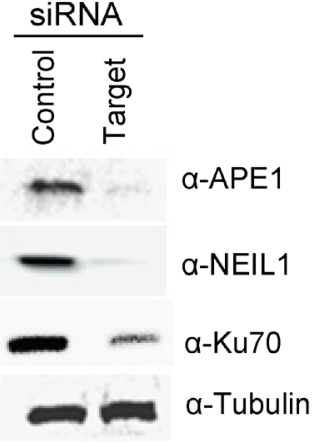

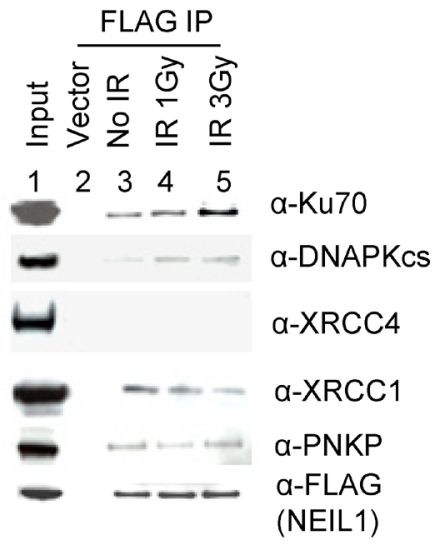

C

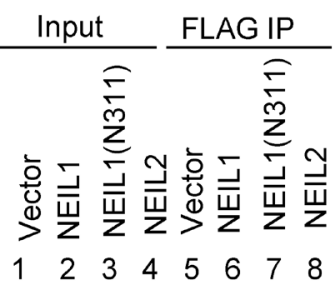

두룽

Mint

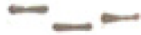

D

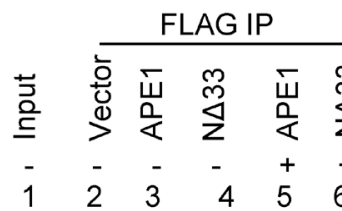

IR (3Gy)

a-Ku70

a-XRCC4

a-PARP1

a-Lig3a

$\alpha$-FLAG

(APE1)

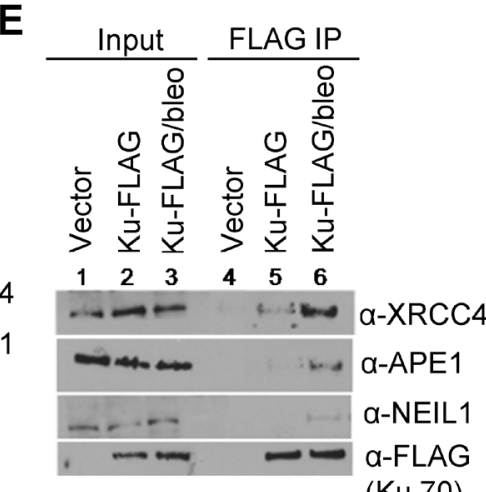

(Ku 70)

F

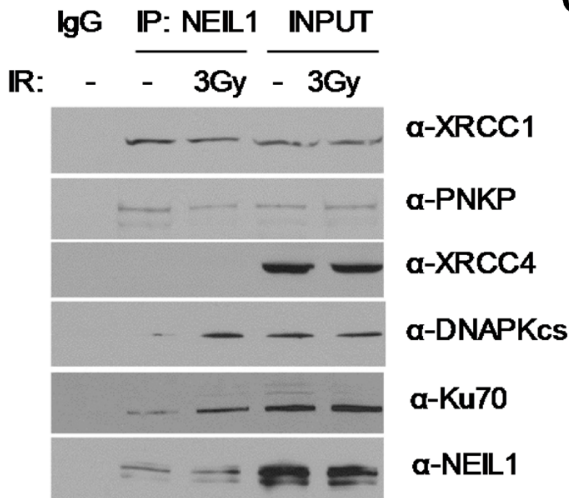

G

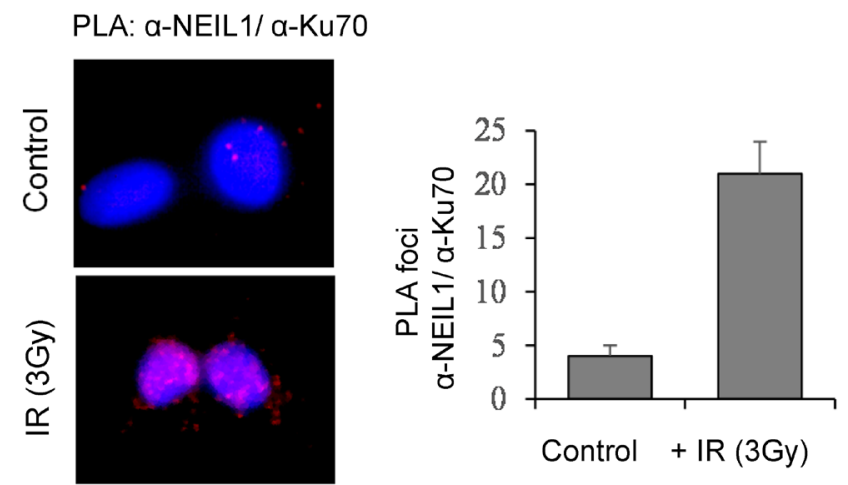

Figure 1: Crosstalk between BER and DSB repair after irradiation. A. Loss of APE1 or NEIL1 causes moderate radiosensitization. For clonogenic survival analysis (left panel), HEK293 cells were irradiated at $48 \mathrm{~h}$ after transfection with siRNAs. Immunoblotting (right panel) shows depletion of target proteins. B. The FLAG-NEIL1 co-IP in HEK293 cells revealed radiation-associated increase in association of Ku, DNA-PKcs, and PNKP but not of XRCC4 with NEIL1. C. The IP of WT APE1 but not of the N $\triangle 33$ mutant (lacking APE1's common interaction domain) contained Ku and BER/SSBR proteins, The FLAG IPs were isolated from HEK293 cells after transfection with FLAGtagged WT APE1 or the N $\triangle 33$ mutant. D. The FLAG-NEIL1/2 IP contained DNA-PKcs and Ku, but the IP of NEIL1(N311) mutant that lacks the common interaction domain (aa312-389) did not contain DNA-PKcs or Ku, underscoring the specificity of Ku interaction. E. The FLAG-Ku IP contained XRCC4, APE1, and NEIL1, whose levels increased after bleomycin treatment. F. Endogenous NEIL1 co-IP contains Ku70, DNA-PKes after irradiation, unlike PNKP and XRCC1, which are constitutively associated. G. PLA analysis confirms incell association of NEIL1 with Ku, which was enhanced after IR treatment ( $>25$ cells were counted for the bar graph). 
IP from HEK293 cells transiently expressing FLAG-Ku provided confirmatory evidence for Ku's interaction with the BER proteins NEIL1 and APE1, as well as the NHEJ protein XRCC4 (Figure 1E). While lack of association of FLAG-NEIL1(N311) polypeptide with $\mathrm{Ku}$ revealed the specificity of interaction, in order to exclude any possible artefacts of ectopic expressions, we further confirmed their in cell association and its enhancement after irradiation by analysing endogenous co-IP with NEIL1 Ab (Figure 1F). Finally, we confirmed our conclusion by using proximity ligation assay (PLA; Figure $1 \mathrm{G}$ ).

\section{Ku inhibits DNA glycosylases and APE1 via direct interaction}

To assess the impact of Ku's interaction with BERinitiating enzymes, we examined their in vitro interaction using purified, recombinant proteins. Affinity co-elution of $\mathrm{Ku}$ with the His-tagged DGs NEIL1, NEIL2, and OGG1, which were pre-bound to Ni-NTA magnetic beads, confirmed Ku's direct binding to the DGs. The absence of $\mathrm{Ku}$ binding to the NEIL1 (N311)-His tag deletion protein was predicted and supports the in-cell data (Figure 2A). With pulldown assay using GSH column bound, GSTtagged NEIL1 CTD peptides aa289-389, aa289-349, aa312-389, aa312-349, or aa350-389, we mapped Ku's interacting region within the CTD of NEIL1. The absence of aa350-389 did not affect binding, which suggests that $\mathrm{Ku}$ binding requires aa289-349 residues of NEIL1 (Figure 2B). Similarly, binary interaction between $\mathrm{Ku}$ and APE1 was confirmed using His and GST pull down assays using His/GST-tagged WT APE1 or the N $\Delta 42$ mutant (Figure 2C).

We then tested the impact of Ku's interaction on the activity of DGs or APE1 using 5-OHU or THF-containing duplex oligonucleotide substrates, respectively, as previously described [35]. The Ku heterodimer inhibited base excision/strand cleavage activity of full-length NEIL1 but not of the N311 mutant in a dose-dependent manner (Figure 2D). This result indicates that inhibition of NEIL1 by $\mathrm{Ku}$ requires its physical interaction with NEIL1. Furthermore, while the dose-dependent NEIL1 inhibition by $\mathrm{Ku}$ was quantitated after 20 min incubation, we confirmed linearity of Ku's inhibition of NEIL1 by measuring activity at 0, 6, 12 and $24 \mathrm{~min}$ (Figure 2E). Similarly, $\mathrm{Ku}$ inhibits other oxidized base-specific DGs NEIL2 and OGG1 as well (Figure 2F). Furthermore, Ku inhibits the 3'dRP lyase activity of APE1 at an SSB site in a duplex oligonucleotide, as well as AP endonuclease activity with a THF-containing substrate (Figure 2G, 2H). In contrast, $\mathrm{Ku}$ did not affect the 3 ' phosphatase activity of polynucleotide kinase-phosphatase (PNKP) at an SSB site (Figure 2G). It should be noted that PNKP is required both in NEIL-initiated BER and NHEJ $[36,37]$.

\section{Distinct association of SAF-A with NHEJ and BER complexes is mediated by SAF-A's DNA-PK- dependent phosphorylation}

SAF-A was previously implicated in the IR-induced genome damage response $[24,25]$ and was shown to be phosphorylated at Ser59 by DNA-PK in irradiated cells. While SAF-A's role in DSB repair was not investigated, we showed earlier that it stimulates NEIL1-initiated BER, and thereby enhances repair of ROS-induced oxidative base damage $[20,38]$. In the present study, we observed association of SAF-A with both NHEJ and BER proteins. Both Ku70 and DNA-PKcs were detected in the SAF-AFLAG IP and their levels were significantly increased after irradiation (Figure 3A). Furthermore, the Ku-SAF-A incell complex reached the peak level at $1 \mathrm{~h}$ post-irradiation (Figure 3B). Next, we examined the phosphorylation status of Ku-bound SAF-A. The FLAG IP from both HEK293 and U2OS cells transiently expressing $\mathrm{Ku}-$ FLAG contained p-Ser59-SAF-A, whose level increased after irradiation (Figure 3C). Kinetic analysis showed the presence of p-Ser59-SAF-A in the Ku-FLAG IP as early as $15 \mathrm{~min}$ post-irradiation, which peaked at $1 \mathrm{~h}$ before returning to the basal level at $4 \mathrm{~h}$ (Figure 3D). These results strongly suggest that phosphorylation of SAF-A regulates its interaction with $\mathrm{Ku}$. When FLAG-tagged phosphomimetic S59D and non-phosphorylable S59A mutants of SAF-A were transiently expressed in HEK293 cells, the presence of $\mathrm{Ku}$ was observed in the IP of both WT SAF-A and the S59D mutant, but not in that of the S59A mutant. These results further support the conclusion that SAF-A, only when phosphorylated, stably associates with $\mathrm{Ku}$ (Figure 3E). Collectively, these data are consistent with the scenario that SAF-A, phosphorylated by DNA$\mathrm{PK}$ in response to IR-induced DNA damage, remains bound to the NHEJ complex until completion of DSB repair, and is subsequently dephosphorylated.

\section{IR induces SAF-A phosphorylation and blocks BER initiation by transient dissociation of NEIL1 from chromatin}

Because SAF-A interacts with both NHEJ and BER proteins $[19,20]$, we explored its possible involvement in the pathways for repair of clustered damage in chromatin. NHEJ of DSBs directly induced by IR occurs in the 15-60 min window after irradiation, as indicated by an increase in 53BP1 level in the chromatin (Figure 4C), consistent with the previous studies [4]. NEIL1's transient dissociation from chromatin was observed in both U2OS and HEK293 cells at 15-30 min post irradiation, at about the same time when SAF-A was phosphorylated (Figure 4A-4C). NEIL1 is restored to chromatin at $1 \mathrm{~h}$ after irradiation when DSB repair is mostly completed, as further revealed by 53BP1 level. This suggests a causal link between SAF-A 
A

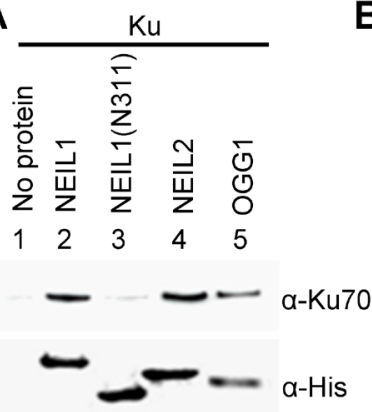

D

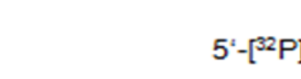

(5-OHU)
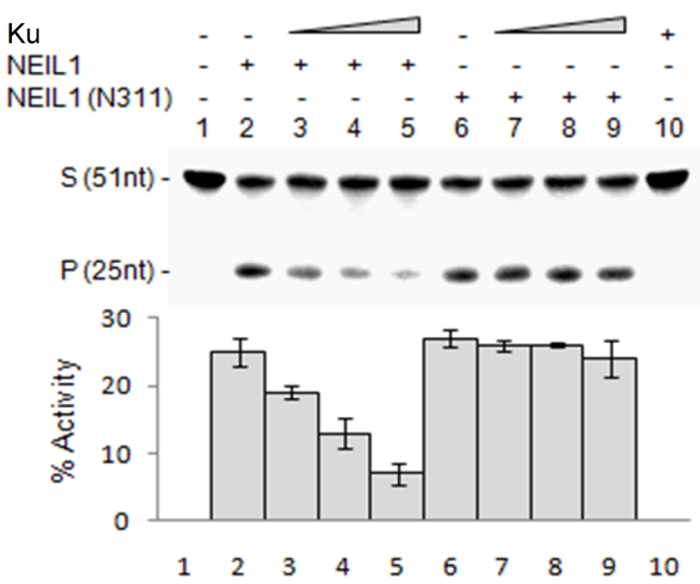

G SSB substrate with $3^{\prime} \mathrm{dRP}$ or $3^{\prime} \mathrm{P}$ ends

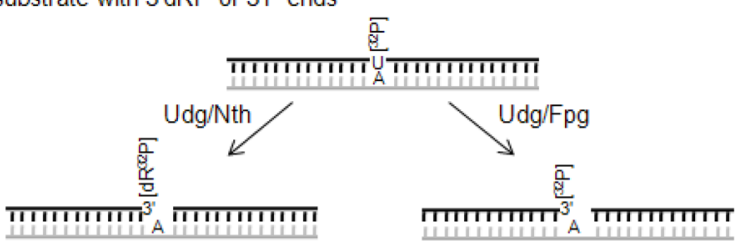

C

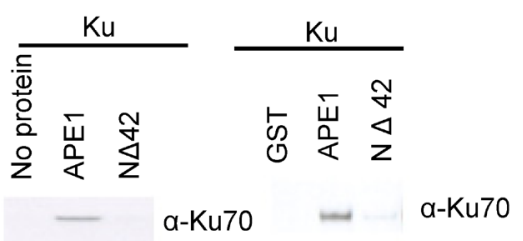

a-Ku70

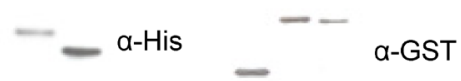

a-GST

E

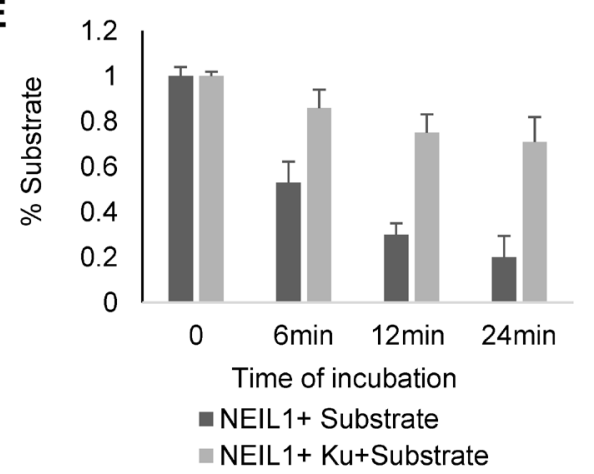

F
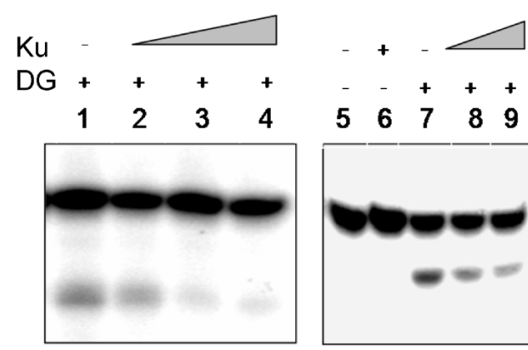

NEIL2

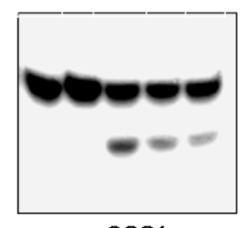

OGG1

3'dRP lyase activity of APE1 3'phosphatase activity of PNKP
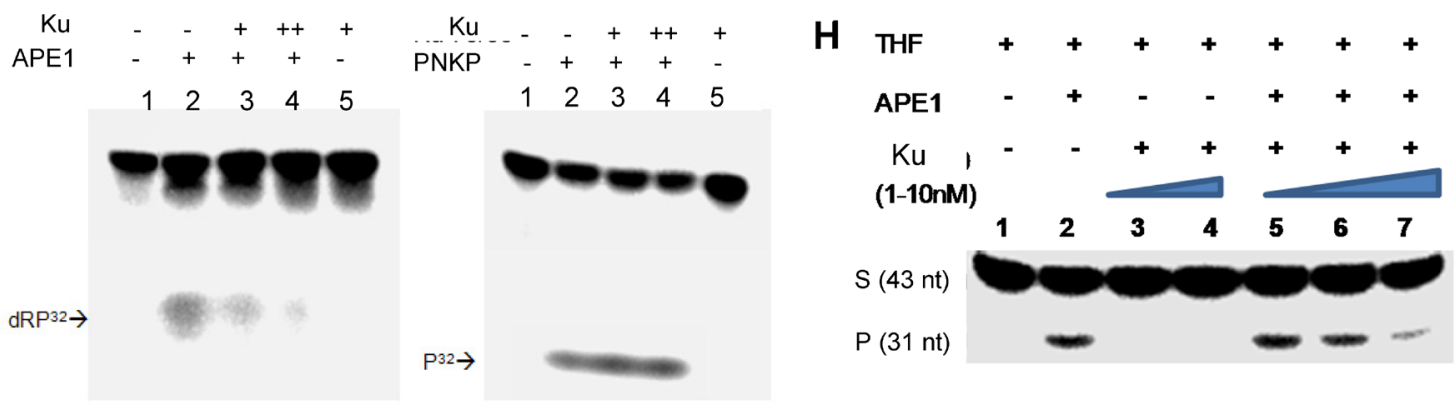

Figure 2: Ku inhibits DGs via direct interaction. A. Direct interaction of Ku with NEIL1 (but not the N311 mutant), NEIL2, and OGG1 was confirmed by His-affinity pull down analysis using purified proteins in the absence of DNA. B. GST pull down analysis showing that NEIL1 aa289-349, but not aa350-389, was needed for Ku binding. C. The binary interaction of Ku with APE1 (but not the $\mathrm{N} \Delta 42$ mutant) is shown by His-affinity pull down analysis. D. Dose-dependent inhibition of WT NEIL1 (1nM, lane 2$)$ by Ku $(0.1,0.5$ and $1 \mathrm{nM}$; lanes 3-5), but not by the N311 mutant (1nM, lane 6-9) is shown with a 5-OHU-containing 51-mer duplex substrate (25nM). E. Time kinetics of Ku inhibition of NEIL1 activity. F. NEIL2 and OGG1 are also inhibited by Ku at similar experimental conditions. G. A 26-nt oligo containing $\mathrm{U}$ at the 5' terminus was labelled with $\gamma^{32} \mathrm{P}-\mathrm{ATP}$ using T4-PNK and annealed with a 25-nt proximal sequence and a 51-nt complementary oligo. The duplex was digested with Udg/Nth or Udg/Fpg to generate a strand break with a 3'dRP or 3'P end, respectively (top). Ku inhibits 3'dRPase activity of APE1 (bottom left panel), but not the 3'phosphatase activity of PNKP (bottom right panel). H. Ku inhibition of AP endonuclease activity of APE1. 
phosphorylation and NEIL1's dissociation from chromatin, in order to prevent BER initiation and allow DSB repair by NHEJ. To further support this possibility, HEK293 cells were treated with the DNA-PK inhibitor NU7441, and the chromatin fraction was analysed at various times after irradiation (Figure 4C). Dissociation of NEIL1 from chromatin was prevented in DNA-PK inhibited cells at 30 min post irradiation. Furthermore, in HEK293 cells with 3'UTR-specific siRNA mediated knockdown of endogenous SAF-A together with ectopic expression of WT SAF-A or the non-phosphorylable mutant, release of chromatin-bound NEIL1 was observed only in WT cells (Figure 4D). It is important to point out that while NEIL1's dissociation from chromatin fraction tightly correlated with SAF-A phosphorylation, peaking at $30 \mathrm{~min}$ after irradiation, NEIL1 re-association with chromatin occurred at $1 \mathrm{~h}$ post IR but SAF-A dephosphorylation followed a slower kinetics, completing after 2-4h. This suggests the presence of additional mechanisms regulating NEIL1's re-association with chromatin. Our recent data show that NEIL1 is acetylated by p300, which is required for its chromatin binding. Here we show that kinetics of NEIL1's dissociation from chromatin post-irradiation correlates with its deacetylation (Supplemental Figure S1). Thus it is likely that NEIL1 acetylation along with other factors are also involved in this regulation.

Additionally, PLA analysis showed only modest increase in NEIL1's in-cell interaction with SAF-A in HEK293 cells at 30 min after irradiation. In contrast, significantly higher number of PLA foci were observed in HEK293 cells expressing FLAG-SAF-A(S59A), suggesting its elevated association with NEIL1 (Figure 4E). These data are consistent with non-phosphorylated SAF-A's higher affinity for NEIL1 (Figure 5B) and lower affinity for $\mathrm{Ku}$ (Figure 3E). These results thus provide strong support for temporal regulation of NHEJ and BER after treatment with IR, coordinated by SAF-A phosphorylation and regulating release of chromatinbound NEIL1 (Figure 4E).

\section{WT SAF-A relieves Ku inhibition of NEIL1 in vitro}

To gain further mechanistic insight about the impact of SAF-A phosphorylation on BER, NEIL1's affinity for

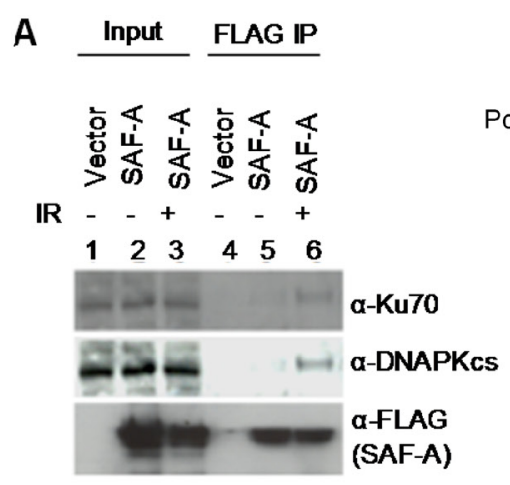

D

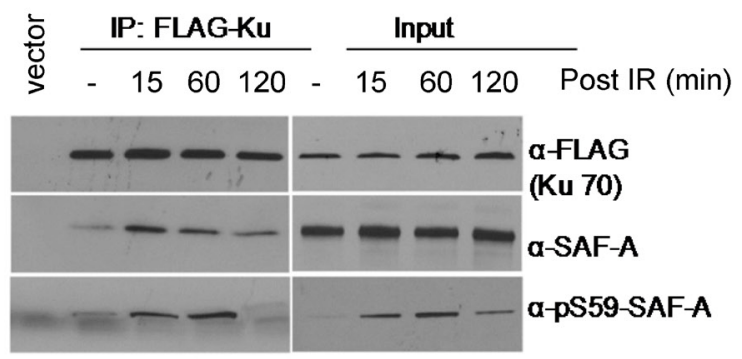

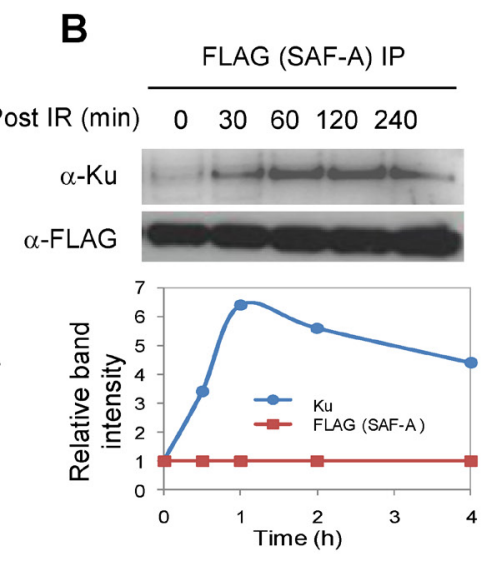

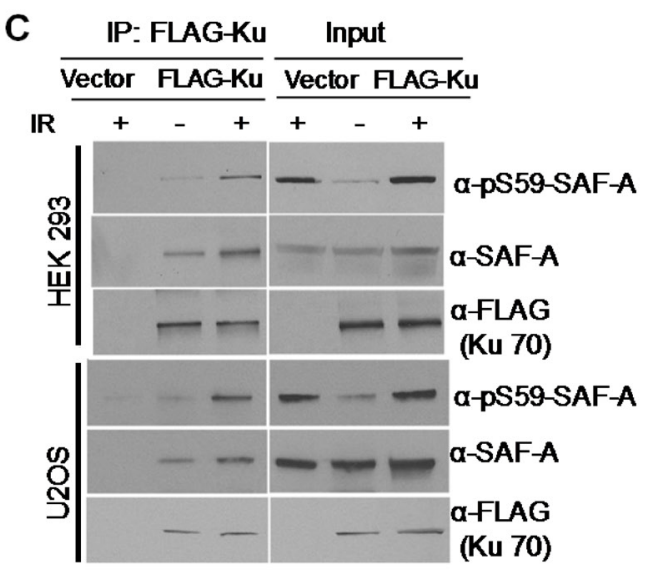

E

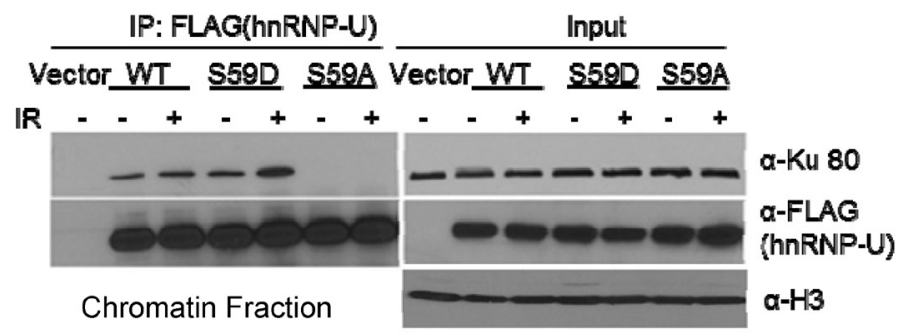

Figure 3: In cell association of Ku with SAF-A is regulated by SAF-A phosphorylation at Ser59. A. Presence of Ku and DNA-PK increased post irradiation (3Gy, 1 h) in the SAF-A IP isolated from HEK293 cells. B. Dynamics of Ku association with SAF-A after irradiation; Ku level was measured in the FLAG-SAF-A IP from HEK293 cells. C. Increased association of SAF-A and pS59-SAF-A in the FLAG-Ku IP after irradiation of HEK293 (upper panel), and U2OS cells (bottom panel). D. Kinetics of association of SAF-A and pS59-SAF-A with FLAG-Ku in HEK293 cells. E. Ku binds to WT SAF-A and the phosphomimetic S59D mutant in chromatin but not to the non-phosphorylatable S59A mutant. 
WT SAF-A versus the S59D mutant was measured by fluorescence and affinity co-elution analyses. In vitro Hisaffinity co-elution of recombinant NEIL1 with His-SAF-A was observed, while co-elution with the S59D mutant was observed at a significantly lower level (Figure 5A). In contrast, $\mathrm{Ku}$ binding to the $\mathrm{S} 59 \mathrm{D}$ mutant was higher compared to the WT SAF-A polypeptide. Fluorescence analysis confirmed this, in which NEIL1's affinity for the WT protein (non-phosphorylated) was found to be about 10-fold higher than for the phosphomimic mutant
(Figure 5B). Together, these results support the scenario that phosphorylated SAF-A, as a component of the NHEJ complex, ensures $\mathrm{Ku}$ inhibition of NEIL1 and may also contribute to NEIL1's dissociation from chromatin in order to prevent BER. SAF-A, dephosphorylated after completion of NHEJ, stimulates NEIL1 even in the presence of $\mathrm{Ku}$, thus acting as a molecular switch for the NHEJ-to-BER transition.

We then investigated the effect of SAF-A phosphorylation on NEIL1's DG activity in vitro. WT
A

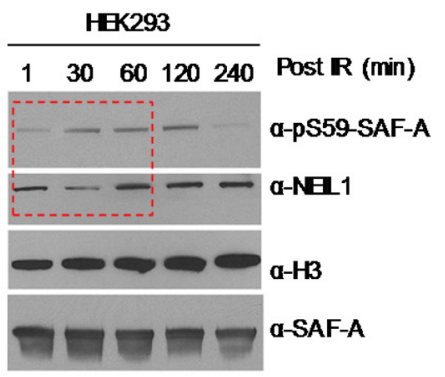

B

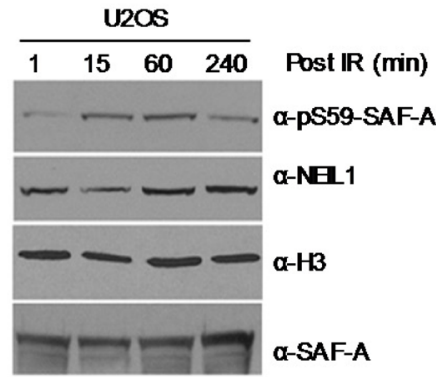

C

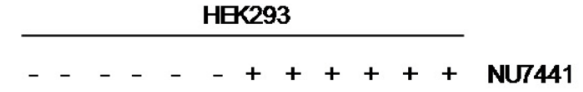

11530601202401153060120240 Post R (min)

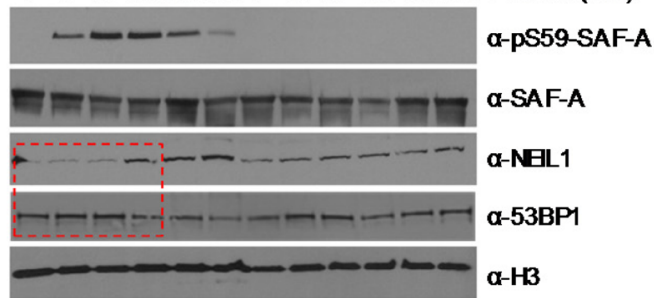

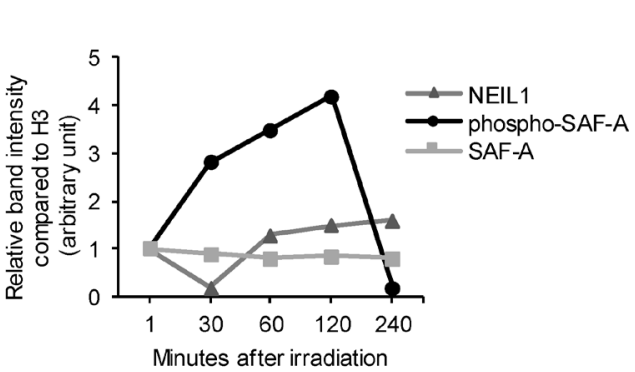

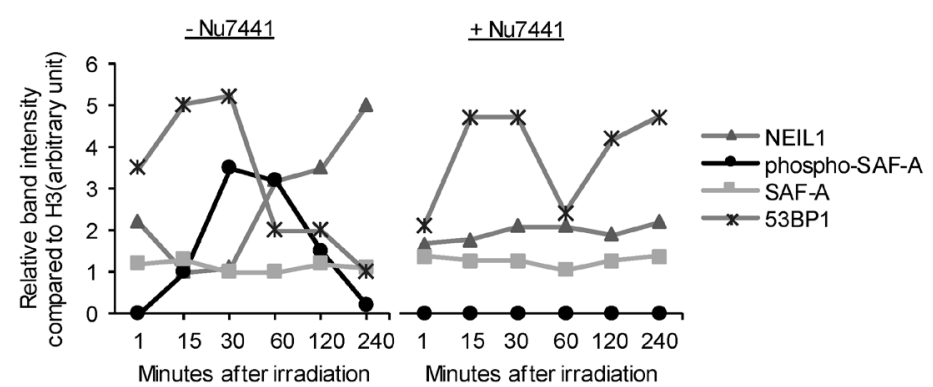

D

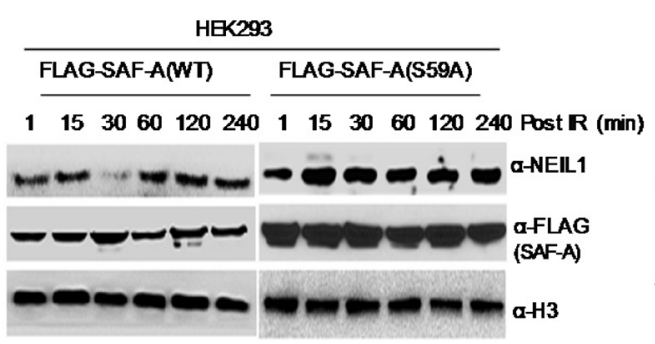

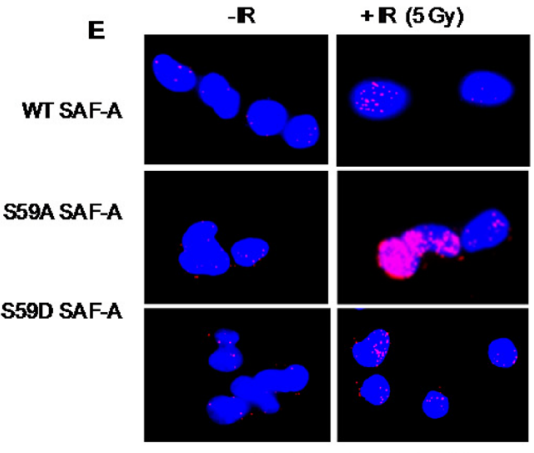

PLA: $\alpha-N B L 1 / \alpha-P L A G(S A F-A)$

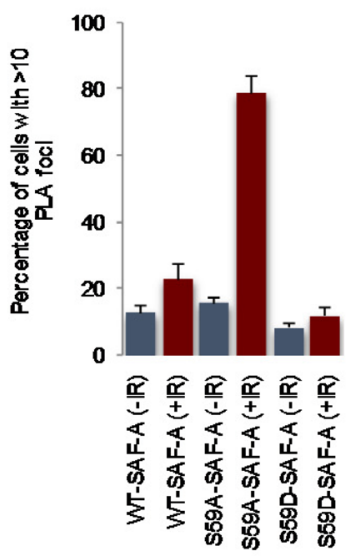

Figure 4: SAF-A phosphorylation by DNA-PK is linked to transient dissociation of NEIL1 from chromatin. A-B. Enhanced pS59 SAF-A formation in the HEK293/ U2OS cell chromatin after irradiation. Chromatin-bound NEIL1 was released at 15$30 \mathrm{~min}$ after IR exposure and restored after 1h, shown in quantitative histogram (lower panel, for HEK293). C. Chromatin-bound NEIL1 level was not altered by pretreatment with the DNA-PKcs inhibitor NU7441, which prevented SAF-A phosphorylation. NU7441 (10 $\mu$ M) pretreatment for $2 \mathrm{~h}$ followed by exposure to $3 \mathrm{~Gy}$ X-rays and chromatin extraction from cells harvested at the indicated times. NEIL1's transient dissociation from chromatin coincided with increase in the 53BP1 level, consistent with NHEJ repair of overt DSBs, while BER was delayed. Quantitative histograms shown in lower panels. D. WT SAF-A, but not the non-phosphorylatable S59A mutant, negatively regulated chromatin-bound NEIL1. HEK293 cells were transfected with 3' UTR hnRNP-U siRNA and FLAG-hnRNP-U WT or S59A, followed by irradiation. E. PLA analysis revealed increased interaction of SAF-A(S59A), but not the WT or S59D mutant with NEIL1 after irradiation. HEK293 cells were transfected with FLAG-tagged WT, S59A, or S59D SAF-A plasmid, and 48 h later, were irradiated (3 Gy). The PLA was performed for NEIL1 and FLAG -SAF-A after further incubation for $30 \mathrm{~min}$. 
SAF-A stimulated NEIL1 activity, confirming earlier studies [20] (Figure 5C), and unlike the S59D mutant overrode $\mathrm{Ku}$ inhibition of NEIL1 (Figure 5D). As $\mathrm{Ku}$ associates with both SAF-A and NEIL1, we next tested whether $\mathrm{Ku}$ forms distinct complex(es) with SAF-A and NEIL1 for its NHEJ and BER role by fractionation of U2OS cell nuclear extracts before and after irradiation, on a Sephacryl-S300 gel filtration column (Supplemental Figure S2). Consistent with our co-IP analysis, Western analysis shows that $\mathrm{Ku}$ elutes in multiple distinct complexes with SAF-A $(>1000 \mathrm{kDa})$ or NEIL1 $(\sim 600 \mathrm{kDa})$.

\section{SAF-A plays a role in IR-induced damage repair and its radioprotective function requires Ser59 phosphorylation}

We previously showed that SAF-A activates NEIL1initiated BER particularly after oxidative stress [20]. Here, we investigated the impact of SAF-A depletion on the repair of IR-induced DSB damage by $\gamma-\mathrm{H} 2 \mathrm{AX}$ foci disappearance and comet analysis. Significant delay in the disappearance of $\gamma-\mathrm{H} 2 \mathrm{AX}$ foci from irradiated (3Gy) U2OS cells was observed after SAF-A depletion compared to that in cells expressing control (scrambled) siRNA (Figure 6A, 6B, 6C). Furthermore, the slower DSB repair kinetics was rescued by ectopic expression of WT SAF-A but not the nonphosphorylatable S59A-

B

Affinity measurements

\begin{tabular}{lc}
\hline Interacting pair & $\mathrm{k}_{\mathrm{D}}(\mathrm{nM})$ \\
\hline & \\
NEIL1+Ku & $584 \pm 37$ \\
NEIL1+SAF-A (WT) & $51 \pm 5.4$ \\
NEIL1+SAF-A (S59D) & $486 \pm 32$ \\
\hline
\end{tabular}

D
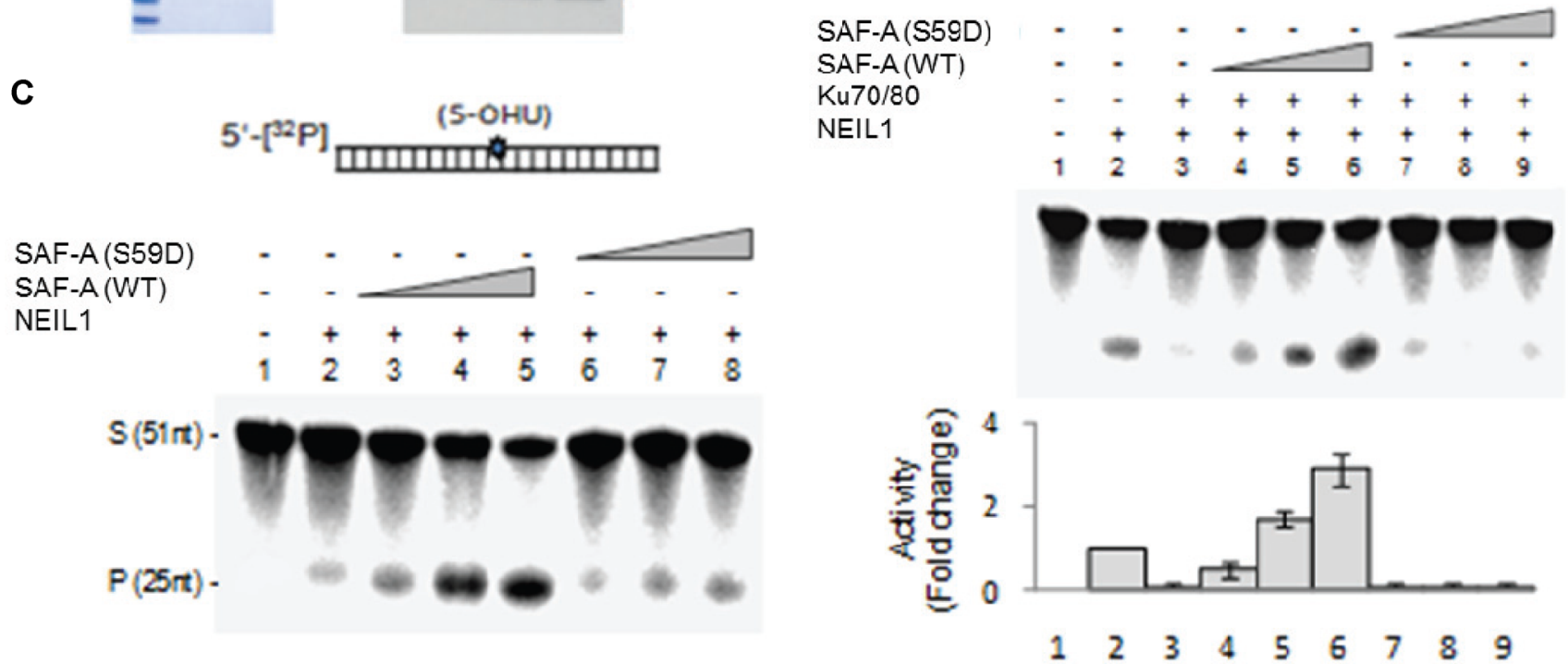

Figure 5: WT SAF-A but not the S59D mutant overrides NEIL1 inhibition by Ku. A. Purification of WT and S59D SAF-A to near homogeneity (left panel after Coomassie staining). In vitro His-pull down of untagged NEIL1 or Ku by His-tagged SAF-A (WT vs. S59D) bound to Ni-beads. NEIL1 binding to the S59D mutant was weaker than binding to WT SAF-A (right panel). B. Affinity measurement of NEIL1 for Ku, WT SAF-A and the S59D mutant by fluorescence analysis where 10-fold lower affinity was observed for the phosphomimetic mutant than for WT SAF-A. C. WT SAF-A (0.1, 05, 1nM; lanes 3-5), but not the S59D mutant (0.1, 05, 1nM; lanes 6-8), stimulated NEIL1 (1nM; lane 2) activity, and D. reversed Ku-dependent inhibition. 
SAF mutant (Figure 6A, 6B, 6C). Consistent with this observation, comet analysis showed comparable level of strand breaks at $4 \mathrm{~h}$ post irradiation in SAF-A depleted cells under alkaline $v s$. neutral condition (Supplemental Figure S3). Furthermore, in view of BER proteins' modest contribution to radioresistance (Figure 1A), we examined the role of SAF-A in cell survival after irradiation. 3'UTR siRNA-mediated depletion of SAF-A caused moderate decrease in clonogenic survival, which was partially rescued by ectopic WT SAF-A, but not by the S59A mutant (Figure 6D). Combined depletion of BER enzymes and SAF-A was then checked for additive sensitivity. Depletion of both NEIL1 and APE1 together with SAF-A caused higher radiosensitivity than depletion of SAF-A alone (Figure 6E). These results are consistent with SAFA's critical role in protection from radiation-induced genome damage.

\section{DISCUSSION}

IR-induced genotoxicity results from the formation of clustered damage in the genome that include overt DSBs together with oxidized base lesions or AP sites, and SSBs. Elucidating the repair processes for IR or radiomimetic drug-induced genome damage is important for improved cancer therapy and protection from accidental or therapeutic radiation. In mammalian cells, the major DSB repair pathways HR and NHEJ are biochemically distinct with diverse substrate requirements, and these follow different kinetics in a cell cycle-dependent manner [39]. NHEJ is the predominant repair pathway for DSBs induced by irradiation or radiomimetic drugs. NHEJdeficient mammalian cells are extremely sensitive to $\mathrm{X}$ and $\gamma$-rays and accumulate unrepaired DSBs as a function of radiation dose [40].
A

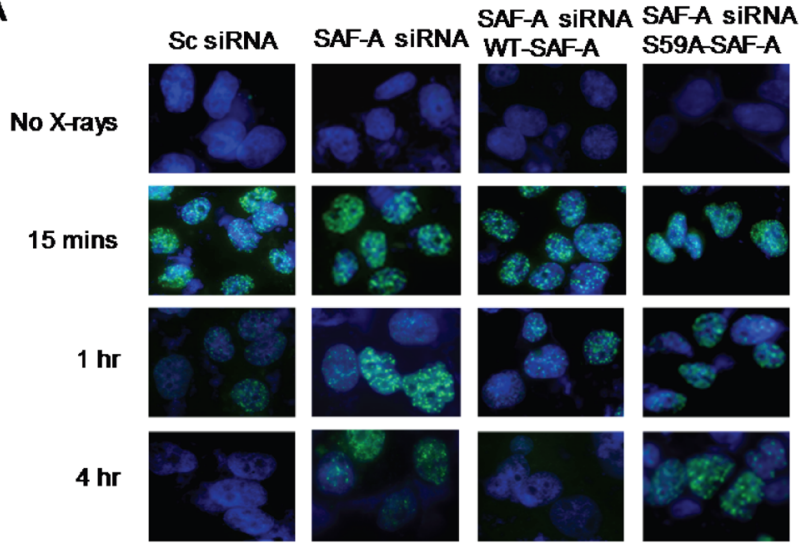

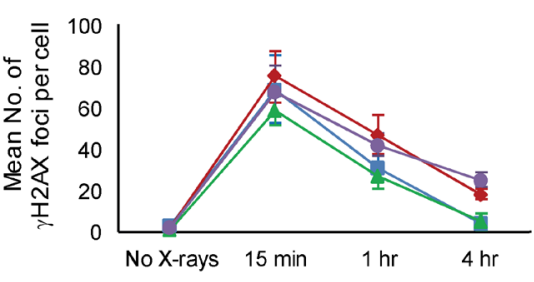

Time after X-ray (3 Gy) treatment - - Sc siRNA

$\longrightarrow$ SAF-A siRNA

- SAF-A SiRNA + SAF-A WT

$\longrightarrow$ SAF-A siRNA + SAF-A S59A
B Control SAF-A SIRNA 3'UTR SIRNA

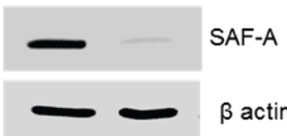

c

FLAG-SAF-A

Vector WT S59A

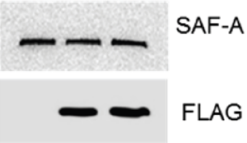

D

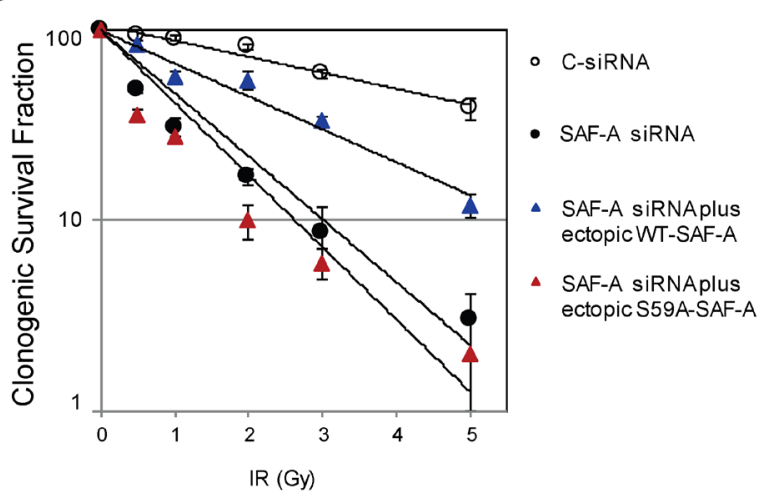

$E$

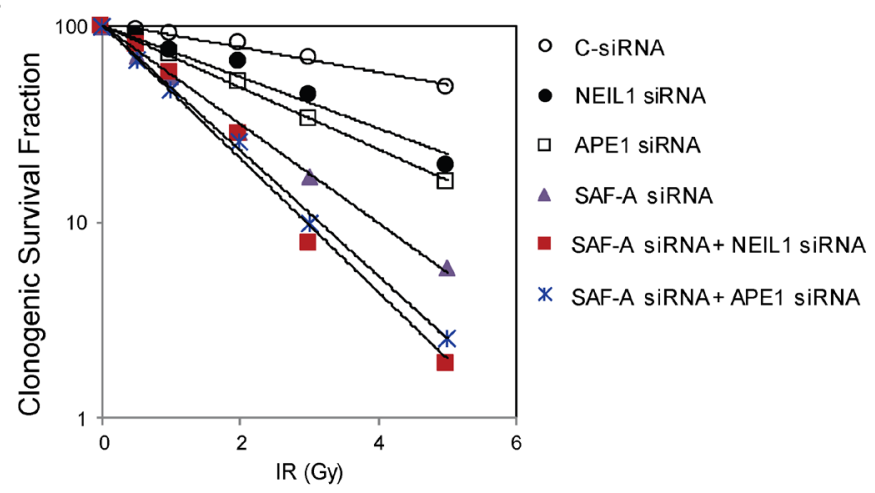

Figure 6: SAF-A's radioprotective function requires its S59 phosphorylation and its additive effect with APE1 and NEIL1. A. HEK293 cells were transfected with 3'UTR-specific SAF-A siRNA alone or in combination with plasmids for WT or S59A FLAG-SAF-A. After irradiated at $48 \mathrm{~h}$ (3Gy), these cells were analysed microscopically for $\gamma \mathrm{H} 2 \mathrm{AX}$ foci kinetics or clonogenic survival analysis. B. Immunoblotting analysis shows downregulation of SAF-A with 3'UTR siRNA in U2OS cells. C. Transient expression of FLAG-SAF-A(WT), FLAG-SAF-A(S59A), FLAG-SAF-A(WT/S59A) in U2OS cells. D. Clonogenic survival of cells transfected with control siRNA, SAF-A 3'UTR siRNA, or SAF-A 3'UTR siRNA plus FLAG-WT/S59A SAF-A plasmids. Cells were irradiated with different dose of x-rays as indicated and three hundred cells were plated in triplicate on 6-cm dishes. After 7-10 days, individual colonies were fixed, stained, and counted for the survival assay. E. SAF-A siRNA-mediated singly or doubly deficient (in combination with NEIL1 or APE1 siRNA) HEK293 cells, along with the control, were irradiated at $48 \mathrm{~h}$ after transfection, and 300 cells were plated in triplicate for the survival assay as before. 
While numerous studies have focused on the repair of IR-induced DSBs by the NHEJ pathway, bi-stranded, non-DSB lesion clusters, which include oxidized bases, SSBs and AP sites that are generated at a much higher level than DSBs, have received little attention. It is likely that the deleterious effects of radiation are primarily caused by clustered damage rather than overt DSBs alone [1]. Earlier studies suggested that overt DSBs in irradiated cells are re-joined first, followed by repair of clustered non-DSB damage at a slower rate [41]. However, how such distinct repair processes are coordinated has not been investigated. As demonstrated in this study and elsewhere [15], non-DSB damage, primarily repaired via the BER/ SSBR pathway, also contributes to the radiosensitivity of tumor cells. Because BER generates intermediate SSBs, repair of IR-induced bi-stranded damage clusters could create additional DSBs and lead to loss of genomic sequences. Furthermore, these additional strand breaks in the vicinity of an overt DSB could cause large deletions $[17,42]$.

Earlier studies suggested that NHEJ alone is not sufficient to handle radiation-induced damage clusters in mammalian cells [41]. Okayasu and his colleagues [43] showed by measuring chromosome fragmentation and $\gamma \mathrm{H} 2 \mathrm{AX}$ foci formation that NHEJ inadequately repairs clustered damage. Recent studies showed that high-energy IR kills more cells than low-energy IR at the same dose level because of inefficient $\mathrm{Ku}$-dependent NHEJ repair, which was subsequently confirmed in NHEJ-deficient mice [44].

The mechanisms of crosstalk between BER and NHEJ at the damage clusters, to prevent large loss of DNA sequences, is not known. We show in this study that coordinated NHEJ repair of overt DSBs precedes repair of neighboring oxidized bases in irradiated cells. The sequential NHEJ $\rightarrow$ BER model (Figure 7) is supported

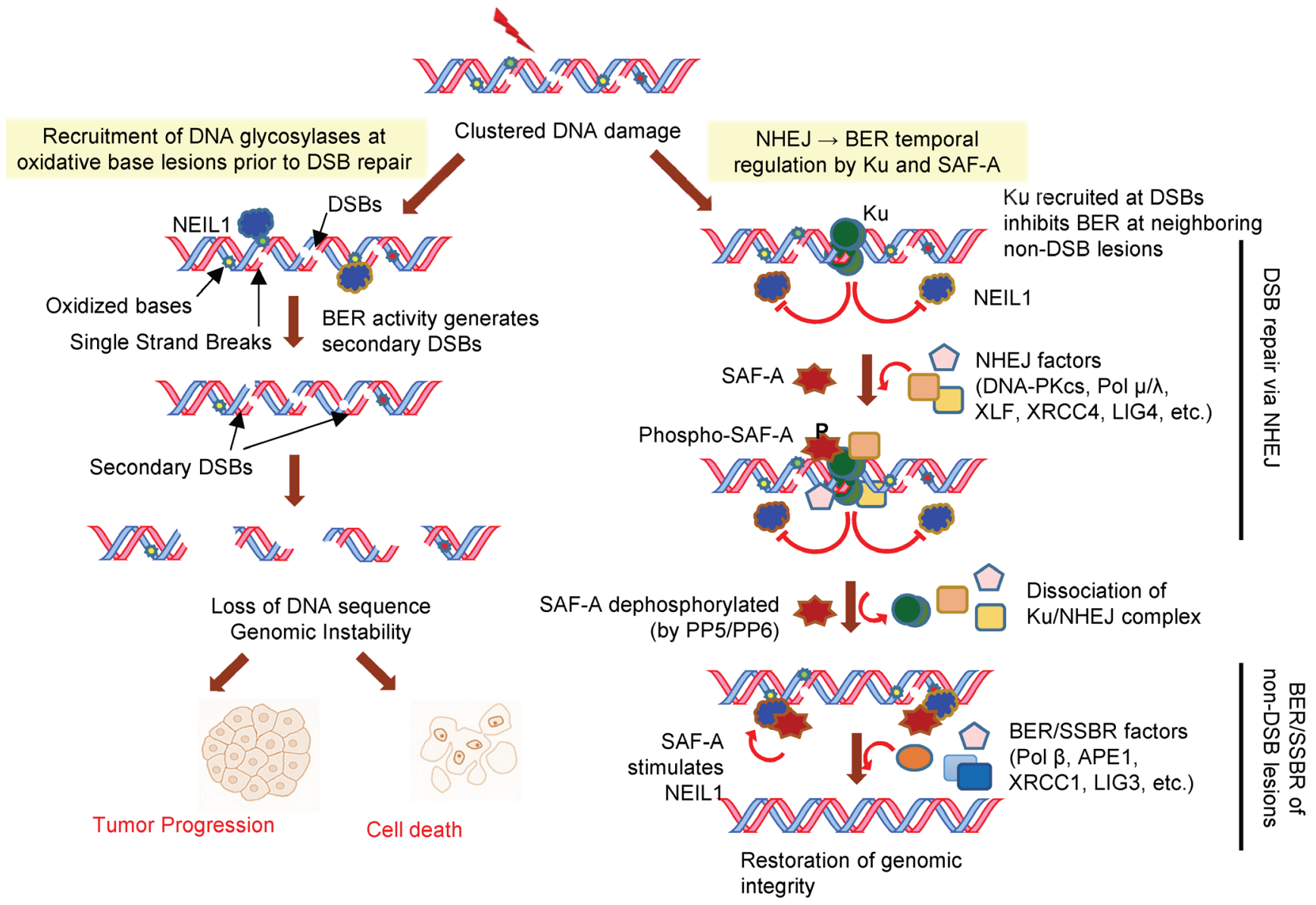

Figure 7: Model of temporal regulation of IR-induced clustered damage in human genome. BER activity at oxidative base lesions at clustered DNA damage could lead to generation of secondary DSBs. Such secondary DSBs generated prior to repair of existing DSBs could lead to loss of genomic sequence. Repair of DSBs in the IR-induced damage cluster is initiated by Ku recruited at DSB, which then assembles the NHEJ complex after recruiting DNA-PKcs. Recruitment of Ku at DSBs also inhibits BER of oxidized bases and AP sites (this study). Early phosphorylation of SAF-A at Ser59 by DNA-PK correlates with transient dissociation of NEIL1 from chromatin to prevent BER initiation. Residual DGs in chromatin are inhibited by Ku while NHEJ occurs. After completion of DSBR, SAF-A is dephosphorylated, relieving Ku inhibition of BER, and restoring NEIL1 levels in chromatin. Ku together with SAF-A thus act as a molecular switch for NHEJ $\rightarrow$ BER transition. 
by three key observations: (a) $\mathrm{Ku}$ immunocomplexes in human cells contain BER proteins including DGs and APE1, all of which are directly inhibited by Ku via binary interaction. This observation is consistent with prior reports of Alt-NHEJ suppression by DNA-PK/Ku [11, $45,46]$. (b) BER inhibition by $\mathrm{Ku}$ is alleviated by SAF-A during NHEJ, but not by the phosphorylated protein $[24,25]$. It is thus likely that after NHEJ completion, SAF-A acts as a molecular switch for the NHEJ $\rightarrow$ BER transition. (c) Consistent with this model, SAF-A via its S59 phosphorylation, regulates transient dissociation of chromatin-bound NEIL1 soon after irradiation, presumably to prevent BER initiation and also allow overt DSB joining via NHEJ. Prevention of dissociation of chromatin-bound NEIL1 by DNA PK inhibition, SAF-A depletion or ectopic non-phosphorylatable S59A mutant, supports this scenario. Furthermore, release of chromatin-bound NEIL1 correlates well with the kinetics of SAF-A phosphorylation and NHEJ of overt DSBs after IR exposure [4, 47], suggesting tight regulation. However, while the NEIL1 level in chromatin is restored $1 \mathrm{~h}$ after IR, SAF-A dephosphorylation requires 2-4 h, suggesting the recovery may involve additional factors or mechanisms.

Once overt DSBs are repaired via NHEJ, repair of non-DSB lesions via BER/SSBR may be initiated, which involves PARP-1 and XRCC1 [4]. Additional DSBs generated during the repair of bi-stranded damage clusters [14-18] are likely repaired exclusively by Alt-EJ because the presence of PARP-1 inhibits Ku recruitment and thus NHEJ $[4,48]$. Consequently, the hierarchy of NHEJ $\rightarrow$ BER/Alt-EJ prevents greater loss of genomic sequences that would otherwise occur with concurrently active BER and NHEJ.

Our studies thus not only underscore the contribution of BER to radioresistance of human cells, but also establish the critical importance of temporal regulation of NHEJ and BER. Because of the extensive use of IR and radiomimetic drugs in cancer therapy, these results may help to identify novel BER/Alt-NHEJ targets along with NHEJ targets for simultaneous radiosensitization of tumors.

\section{MATERIALS AND METHODS}

\section{Cell culture}

The human embryonic kidney HEK293 (ATCC \# CRL-1573) and osteosarcoma U2OS (ATCC \# HTB-96) cell lines were grown in Dulbecco's Modified Eagle's medium (DMEM; Gibco, Carlsbad, CA) supplemented with $10 \%$ fetal bovine serum (FBS) and $100 \mu \mathrm{g} / \mathrm{ml}$ penicillin/streptomycin (Thermo Fisher, Waltham, MA) at $37^{\circ} \mathrm{C}$ in the presence of $5 \% \mathrm{CO}_{2}$ and $95 \%$ relative humidity. Zeocin $(100 \mu \mathrm{g} / \mathrm{ml})$ was supplemented in the medium for culturing HEK293 cells stably expressing FLAG-NEIL1.

\section{Subcellular fractionation}

The chromatin fraction was prepared as previously described [49]. Briefly, after harvesting by scraping culture dishes, the cells were lysed in a cytoplasmic buffer [10 mM Tris- $\mathrm{HCl} \mathrm{pH}$ 7.9, $0.34 \mathrm{M}$ sucrose, $3 \mathrm{mM} \mathrm{CaCl}_{2}, 2$ $\mathrm{mM} \mathrm{Mg}(\mathrm{OAc})_{2}, 0.1 \mathrm{mM}$ EDTA, $1 \mathrm{mM}$ DTT, 0.1\% Nonidet $\mathrm{P}-40$, and protease inhibitor mixture (Roche Applied Science)], and then centrifuged at $3500 \times \mathrm{g}$ for $15 \mathrm{~min}$ at $4^{\circ} \mathrm{C}$. The pellet was suspended in nuclear lysis buffer (20 mM HEPES pH 7.9, 1.5 mM $\mathrm{MgCl}_{2}, 3$ mM EDTA, $1 \mathrm{mM}$ DTT, $10 \%$ glycerol, $0.5 \%$ Nonidet P-40, and the protease inhibitor mixture), and centrifuged at $14000 \mathrm{rpm}$ for $10 \mathrm{~min}$ to separate the soluble nuclear fraction from the chromatin pellet. The pellet was dispersed in chromatin lysis buffer (150 mM HEPES pH 7.9, $1.5 \mathrm{mM} \mathrm{MgCl}_{2}$, $150 \mathrm{mM}$ potassium acetate, $10 \%$ glycerol, and protease inhibitor mixture), digested with 0.15 unit/ $\mu$ l of benzonase or EtBR at $37^{\circ} \mathrm{C}$ for $30 \mathrm{~min}$ to degrade or remove nucleic acids, and centrifuged at $14000 \mathrm{rpm}$ for $15 \mathrm{~min}$ at $4^{\circ} \mathrm{C}$. The supernatant chromatin fraction was used for analysis.

\section{Co-immunoprecipitation (Co-IP) assay}

For co-IP assay, the lysates of chromatin or the nuclear lysates were immunoprecipitated for $3 \mathrm{~h}$ at $4^{\circ} \mathrm{C}$ with FLAG M2 Ab-bound agarose beads (Sigma, St. Louis, MO). The beads were washed three times with $0.5 \mathrm{ml}$ cold Tris-buffered saline (TBS), eluted in $40 \mu \mathrm{l}$ Laemmli buffer. The eluate was separated by SDS-PAGE for immunoblotting with appropriate Abs.

\section{Antibodies}

Anti-hnRNP-U/SAF-A, anti-APE1, anti-Ku 70, antiDNA-PKcs, anti-beta tubulin, anti-beta actin, and antiLig3 Abs were purchased from Abcam (Cambridge, MA). Anti-APE1 was obtained from Santa Cruz Biotechnology (Santa Cruz, CA). Anti-PARP1, anti-Ku 80, anti-XRCC1, anti-XRCC4, anti-GST, anti-His, anti-H3, and anti-NBS1 were purchased from Cell Signaling (Beverly, MA). AntiFLAG Ab was purchased from Sigma (St. Louis, MO). The rabbit anti-NEIL1 and anti-pS59 SAF-A Abs were custom-generated $[26,33,50]$.

\section{Purification of recombinant proteins}

Recombinant wild type (WT) human NEIL1, APE1 and their truncation mutants and the truncated polypeptides of NEIL2, OGG1, and WT SAF-A were all purified from Escherichia coli as described previously 
[33]. The expression plasmids for S59D and S59A SAF-A mutant were generated by site-directed mutagenesis (Agilent Technologies). Ku (Ku70/80 heterodimer) and DNA-PKcs were purified from HeLa cells as described previously [51].

\section{In vitro affinity co-elution assay}

For the His-affinity pull down assay, His-tagged WT SAF-A or the S59D mutant was bound to Ni-NTA beads $(20 \mu \mathrm{l})$, mixed with full-length NEIL1 or $\mathrm{Ku}$ in $0.5 \mathrm{ml}$ TBS buffer and incubated with constant rocking for $4 \mathrm{~h}$ at $4^{\circ} \mathrm{C}$. After washing the beads five times with 50 volume TBS buffer, the bound proteins were eluted with SDS/ PAGE loading buffer and fractionated by SDS/PAGE for immunoblotting.

\section{In situ PLA}

HEK293 cells were cultured in 16-well chamber slides and co-transfected with siRNA of WT SAF-A or FLAG-tagged WT SAF-A and its S59A, or S59D mutants. At $48 \mathrm{~h}$ following co-transfection the cells were exposed to $3 \mathrm{~Gy}$ x-rays, were fixed with $4 \%$ paraformaldehyde, permeabilized with $0.2 \%$ Tween 20 , and then incubated with the primary Ab for NEIL1 (rabbit) or FLAG-SAF-A; mouse monoclonal). The PLA assay was performed using the Duolink PLA kit (Olink Bioscience, Uppsala, Sweden) according to the manufacturer's instructions. The PLA signals were visualized in a fluorescence microscope (Olympus) at 200× magnification.

\section{Clonogenic survival assay}

Log phase HEK293 cells were transfected with NEIL1 siRNA (80 nM, targeting the $3^{\prime}$ UTR region of the NEIL1 gene; sense sequence, 5'-CCGUGAUGAUGUUUGUUUAUU-3'; antisense sequence, 5'-UAAACAAACAUCAUCACGGUU-3'; Sigma, St. Louis, MO), SAF-A siRNA (Dharmacon, catalogue number J-013501-05), APE1 siRNA (80nM, cat\#SASI_Hs01_00027147, Sigma), individually or together for $48 \mathrm{~h}$. Scrambled siRNA was used as a control. Downregulation of target genes was confirmed by immunoblotting of the cell extracts $48 \mathrm{~h}$ after transfection. Cells were irradiated with 2 Gy, 4 Gy, and 6 Gy x-rays and transferred to 6-well plates (300 cells/well) in triplicate. After allowing the cells to grow in fresh medium for 7-10 days, the colonies were stained with $0.5 \%$ crystal violet and counted.

\section{Affinity measurement by fluorescence spectroscopy}

Interaction of SAF-A or Ku with NEIL1 C-terminal peptide (residues 312-349 lacking aromatic residues) was analysed from the change in intrinsic tryptophan fluorescence (excitation wavelength 295; emission wavelength $300-450 \mathrm{~nm}$ ) of SAF-A or Ku, using a LS50 spectrofluorometer (PerkinElmer Life Sciences), after incubation in $10 \mathrm{mM}$ PBS pH 7.5 and 5\% glycerol at $25^{\circ} \mathrm{C}$ for $5 \mathrm{~min}$. The binding constant $K_{D}$ was calculated by plotting $\Delta F$ (change in fluorescence at $345 \mathrm{~nm}$ ) versus ligand concentration according to the equation $\Delta F=$ $\Delta F \max \times[$ ligand $] / K_{D}+[$ ligand $][20,52]$.

\section{Analysis of NEIL1, NEIL2, OGG1, APE1, and PNKP activity}

The DNA glycosylase activities of NEIL1, NEL2, or their truncated mutants were assessed using a 5'-32 P-labeled 5-OHU-containing oligonucleotide substrate as previously described $[20,52]$. The OGG1 activity was analysed in a similar manner using an 8-OxoG containing oligo [53]. The 3' phosphatase activity of PNKP, and the AP lyase activities of APE1 and 3'dRP were performed as previously described $[35,54]$.

\section{ACKNOWLEDGMENTS}

This work was supported primarily by the National Institutes of Health USPHS grant R01 CA158910 (S.M.) and in part by R01 CA NS088645 (M.L.H.), R01 GM105090 (S.M.), PO1 CA92584 (S.M. and S.P.L.M), Canadian Institute of Health MOP13639 (S.P.L.M.) and HMRI Institutional Funds (S.M. and M.L.H.). We thank Drs Tapas Hazra and Istvan Boldogh at University of Texas Medical Branch, Galveston for critical reading of the manuscript and A. Kazi at HMRI for assistance in quantitation analysis.

\section{Author contributions}

SM and MLH conceived and co-ordinated the research and wrote the paper with contributions from AD, CY, AKM, PMH, AP, SS, YY, DC, PC and SPLM. Experiments were performed by MLH, AD, CY, AKM, $\mathrm{PMH}, \mathrm{AP}, \mathrm{SSG}$ and YY. DC provided recombinant $\mathrm{Ku}$ and DNA-PKcs for in vitro studies. SPLM and YY provided Ab to P-Ser59 SAF-A, which was also generated by PC.

\section{CONFLICTS OF INTERESTS}

The authors declare no competing financial interests. 


\section{SIGNIFICANCE}

Elucidating the repair processes for ionizing radiation (IR)- or radiomimetic drug-induced genome damage should be important for improving cancer therapy as well as protection of healthy tissues. IR induces DNA double strand breaks (DSBs) together with high levels of oxidized bases and single-strand breaks (SSBs) in close proximity, which requires multiple repair pathways to restore genomic integrity. Here, we have identified hierarchical repair where non-homologous end-joining (NHEJ)-mediated DSB repair precedes repair of oxidized bases. Such temporal regulation results from transient inhibition of base repair by the NHEJ protein Ku. IRinduced phosphorylation of SAF-A/hnRNP-U regulates $\mathrm{Ku}$ 's inhibitory function, which may be critical for preventing loss of DNA fragments, and could be explored for enhancing tumor radiosensitivity.

\section{Editorial note}

This paper has been accepted based in part on peerreview conducted by another journal and the authors' response and revisions as well as expedited peer-review in Oncotarget.

\section{REFERENCES}

1. Eccles LJ, O’Neill P and Lomax ME. Delayed repair of radiation induced clustered DNA damage: friend or foe? Mutat Res. 2011; 711(1-2):134-141.

2. Sage E and Harrison L. Clustered DNA lesion repair in eukaryotes: relevance to mutagenesis and cell survival. Mutat Res. 2011; 711(1-2):123-133.

3. Ciccia A and Elledge SJ. The DNA damage response: making it safe to play with knives. Mol Cell. 2010; 40(2):179-204.

4. Mahaney BL, Meek K and Lees-Miller SP. Repair of ionizing radiation-induced DNA double-strand breaks by non-homologous end-joining. Biochem J. 2009; 417(3):639650 .

5. Lobrich $\mathrm{M}$ and Jeggo PA. Harmonising the response to DSBs: a new string in the ATM bow. DNA Repair (Amst). 2005; 4(7):749-759.

6. Dobbs TA, Tainer JA and Lees-Miller SP. A structural model for regulation of NHEJ by DNAPKcs autophosphorylation. DNA Repair (Amst). 2010; 9(12):1307-1314.

7. Lieber MR. The mechanism of double-strand DNA break repair by the nonhomologous DNA end-joining pathway. Annu Rev Biochem. 2010; 79:181-211.

8. Hegde ML, Hazra TK and Mitra S. Early steps in the DNA base excision/single-strand interruption repair pathway in mammalian cells. Cell Res. 2008; 18(1):27-47.
9. Caldecott KW. Single-strand break repair and genetic disease. Nat Rev Genet. 2008; 9(8):619-631.

10. Davis AJ and Chen DJ. DNA double strand break repair via non-homologous end-joining. Transl Cancer Res. 2013; 2(3):130-143.

11. Rassool FV and Tomkinson AE. Targeting abnormal DNA double strand break repair in cancer. Cell Mol Life Sci. 2010; 67(21):3699-3710.

12. Sutherland BM, Bennett PV, Sidorkina O and Laval J. Clustered damages and total lesions induced in DNA by ionizing radiation: oxidized bases and strand breaks. Biochemistry. 2000; 39(27):8026-8031.

13. Blaisdell JO and Wallace SS. Abortive base-excision repair of radiation-induced clustered DNA lesions in Escherichia coli. Proc Natl Acad Sci U S A. 2001; 98(13):7426-7430.

14. Yang N, Chaudhry MA and Wallace SS. Base excision repair by hNTH1 and hOGG1: a two edged sword in the processing of DNA damage in gamma-irradiated human cells. DNA Repair (Amst). 2006; 5(1):43-51.

15. Yang N, Galick $H$ and Wallace SS. Attempted base excision repair of ionizing radiation damage in human lymphoblastoid cells produces lethal and mutagenic double strand breaks. DNA Repair (Amst). 2004; 3(10):1323-1334.

16. Georgakilas AG, Bennett PV, Wilson DM, 3rd and Sutherland BM. Processing of bistranded abasic DNA clusters in gamma-irradiated human hematopoietic cells. Nucleic Acids Res. 2004; 32(18):5609-5620.

17. Gulston M, de Lara C, Jenner T, Davis E and O'Neill P. Processing of clustered DNA damage generates additional double-strand breaks in mammalian cells post-irradiation. Nucleic Acids Res. 2004; 32(4):1602-1609.

18. Paap B, Wilson DM, 3rd and Sutherland BM. Human abasic endonuclease action on multilesion abasic clusters: implications for radiation-induced biological damage. Nucleic Acids Res. 2008; 36(8):2717-2727.

19. Banerjee D, Mandal SM, Das A, Hegde ML, Das S, Bhakat KK, Boldogh I, Sarkar PS, Mitra S and Hazra TK. Preferential repair of oxidized base damage in the transcribed genes of mammalian cells. J Biol Chem. 2011; 286(8):6006-6016.

20. Hegde ML, Banerjee S, Hegde PM, Bellot LJ, Hazra TK, Boldogh I and Mitra S. Enhancement of NEIL1 protein-initiated oxidized DNA base excision repair by heterogeneous nuclear ribonucleoprotein U (hnRNP-U) via direct interaction. J Biol Chem. 2012; 287(41):3420234211.

21. Xiao R, Tang P, Yang B, Huang J, Zhou Y, Shao C, Li H, Sun $\mathrm{H}$, Zhang $\mathrm{Y}$ and $\mathrm{Fu} \mathrm{XD}$. Nuclear matrix factor hnRNP U/SAF-A exerts a global control of alternative splicing by regulating U2 snRNP maturation. Mol Cell. 2012; 45(5):656-668.

22. Romig H, Fackelmayer FO, Renz A, Ramsperger U and Richter A. Characterization of SAF-A, a novel nuclear DNA binding protein from HeLa cells with high affinity for 
nuclear matrix/scaffold attachment DNA elements. Embo j. 1992; 11(9):3431-3440.

23. Spraggon L, Dudnakova T, Slight J, Lustig-Yariv O, Cotterell J, Hastie N and Miles C. hnRNP-U directly interacts with WT1 and modulates WT1 transcriptional activation. Oncogene. 2007; 26(10):1484-1491.

24. Britton S, Froment C, Frit P, Monsarrat B, Salles B and Calsou P. Cell nonhomologous end joining capacity controls SAF-A phosphorylation by DNA-PK in response to DNA double-strand breaks inducers. Cell Cycle. 2009; 8(22):3717-3722.

25. Berglund FM and Clarke PR. hnRNP-U is a specific DNAdependent protein kinase substrate phosphorylated in response to DNA double-strand breaks. Biochem Biophys Res Commun. 2009; 381(1):59-64.

26. Douglas P, Ye R, Morrice N, Britton S, Trinkle-Mulcahy L and Lees-Miller SP. Phosphorylation of SAF-A/hnRNP-U Serine 59 by Polo-Like Kinase 1 Is Required for Mitosis. Mol Cell Biol. 2015; 35(15):2699-2713.

27. O'Driscoll M and Jeggo PA. The role of double-strand break repair - insights from human genetics. Nat Rev Genet. 2006; 7(1):45-54.

28. Meek K, Gupta S, Ramsden DA and Lees-Miller SP. The DNA-dependent protein kinase: the director at the end. Immunol Rev. 2004; 200:132-141.

29. Rosenquist TA, Zaika E, Fernandes AS, Zharkov DO, Miller H and Grollman AP. The novel DNA glycosylase, NEIL1, protects mammalian cells from radiation-mediated cell death. DNA Repair (Amst). 2003; 2(5):581-591.

30. Batuello CN, Kelley MR and Dynlacht JR. Role of Ape1 and base excision repair in the radioresponse and heatradiosensitization of HeLa Cells. Anticancer Res. 2009; 29(4):1319-1325.

31. Suhasini AN, Sommers JA, Mason AC, Voloshin ON, Camerini-Otero RD, Wold MS and Brosh RM, Jr. FANCJ helicase uniquely senses oxidative base damage in either strand of duplex DNA and is stimulated by replication protein A to unwind the damaged DNA substrate in a strand-specific manner. J Biol Chem. 2009; 284(27):1845818470 .

32. Hegde ML, Hegde PM, Arijit D, Boldogh I and Mitra S. Human DNA Glycosylase NEIL1's Interactions with Downstream Repair Proteins Is Critical for Efficient Repair of Oxidized DNA Base Damage and Enhanced Cell Survival. Biomolecules. 2012; 2(4):564-578.

33. Hegde ML, Hegde PM, Bellot LJ, Mandal SM, Hazra TK, Li GM, Boldogh I, Tomkinson AE and Mitra S. Prereplicative repair of oxidized bases in the human genome is mediated by NEIL1 DNA glycosylase together with replication proteins. Proc Natl Acad Sci U S A. 2013; 110(33):E3090-3099.

34. Bhakat KK, Mantha AK and Mitra S. Transcriptional regulatory functions of mammalian AP-endonuclease
(APE1/Ref-1), an essential multifunctional protein. Antioxid Redox Signal. 2009; 11(3):621-638.

35. Mantha AK, Oezguen N, Bhakat KK, Izumi T, Braun W and Mitra S. Unusual role of a cysteine residue in substrate binding and activity of human AP-endonuclease 1. J Mol Biol. 2008; 379(1):28-37.

36. Zolner AE, Abdou I, Ye R, Mani RS, Fanta M, Yu Y, Douglas P, Tahbaz N, Fang S, Dobbs T, Wang C, Morrice N, Hendzel MJ, Weinfeld M and Lees-Miller SP. Phosphorylation of polynucleotide kinase/ phosphatase by DNA-dependent protein kinase and ataxia-telangiectasia mutated regulates its association with sites of DNA damage. Nucleic Acids Res. 2011; 39(21):9224-9237.

37. Mani RS, Yu Y, Fang S, Lu M, Fanta M, Zolner AE, Tahbaz N, Ramsden DA, Litchfield DW, Lees-Miller SP and Weinfeld M. Dual modes of interaction between XRCC4 and polynucleotide kinase/phosphatase: implications for nonhomologous end joining. J Biol Chem. 2010; 285(48):37619-37629.

38. Dutta A, Yang C, Sengupta S, Mitra S and Hegde ML. New paradigms in the repair of oxidative damage in human genome: mechanisms ensuring repair of mutagenic base lesions during replication and involvement of accessory proteins. Cell Mol Life Sci. 2015.

39. Tamulevicius P, Wang M and Iliakis G. Homology-directed repair is required for the development of radioresistance during $\mathrm{S}$ phase: interplay between double-strand break repair and checkpoint response. Radiat Res. 2007; 167(1):111.

40. Nagasawa H, Little JB, Inkret WC, Carpenter S, Raju MR, Chen DJ and Strniste GF. Response of X-ray-sensitive CHO mutant cells (xrs-6c) to radiation. II. Relationship between cell survival and the induction of chromosomal damage with low doses of alpha particles. Radiat Res. 1991; 126(3):280-288.

41. Asaithamby A, Hu B and Chen DJ. Unrepaired clustered DNA lesions induce chromosome breakage in human cells. Proc Natl Acad Sci U S A. 2011; 108(20):8293-8298.

42. Singleton BK, Griffin CS and Thacker J. Clustered DNA damage leads to complex genetic changes in irradiated human cells. Cancer Res. 2002; 62(21):6263-6269.

43. Okayasu R, Okada M, Okabe A, Noguchi M, Takakura $\mathrm{K}$ and Takahashi S. Repair of DNA damage induced by accelerated heavy ions in mammalian cells proficient and deficient in the non-homologous end-joining pathway. Radiat Res. 2006; 165(1):59-67.

44. Wang H, Wang X, Zhang P and Wang Y. The Ku-dependent non-homologous end-joining but not other repair pathway is inhibited by high linear energy transfer ionizing radiation. DNA Repair (Amst). 2008; 7(5):725-733.

45. Fattah F, Lee EH, Weisensel N, Wang Y, Lichter N and Hendrickson EA. Ku regulates the non-homologous end joining pathway choice of DNA double-strand break repair 
in human somatic cells. PLoS Genet. 2010; 6(2):e1000855.

46. Perrault R, Wang H, Wang M, Rosidi B and Iliakis G. Backup pathways of NHEJ are suppressed by DNA-PK. J Cell Biochem. 2004; 92(4):781-794.

47. Gupta A, Hunt CR, Hegde ML, Chakraborty S, Udayakumar D, Horikoshi N, Singh M, Ramnarain DB, Hittelman WN, Namjoshi S, Asaithamby A, Hazra TK, Ludwig T, Pandita RK, Tyler JK and Pandita TK. MOF phosphorylation by ATM regulates 53BP1-mediated double-strand break repair pathway choice. Cell Rep. 2014; 8(1):177-189.

48. Wang M, Wu W, Wu W, Rosidi B, Zhang L, Wang H and Iliakis G. PARP-1 and $\mathrm{Ku}$ compete for repair of DNA double strand breaks by distinct NHEJ pathways. Nucleic Acids Res. 2006; 34(21):6170-6182.

49. Aygun O, Svejstrup J and Liu Y. A RECQ5-RNA polymerase II association identified by targeted proteomic analysis of human chromatin. Proc Natl Acad Sci U S A. 2008; 105(25):8580-8584.

50. Hazra TK, Izumi T, Boldogh I, Imhoff B, Kow YW, Jaruga P, Dizdaroglu M and Mitra S. Identification and characterization of a human DNA glycosylase for repair of modified bases in oxidatively damaged DNA. Proc Natl Acad Sci U S A. 2002; 99(6):3523-3528.
51. Goodarzi AA and Lees-Miller SP. Biochemical characterization of the ataxia-telangiectasia mutated (ATM) protein from human cells. DNA Repair (Amst). 2004; 3(7):753-767.

52. Hegde ML, Theriot CA, Das A, Hegde PM, Guo Z, Gary RK, Hazra TK, Shen B and Mitra S. Physical and functional interaction between human oxidized base-specific DNA glycosylase NEIL1 and flap endonuclease 1. J Biol Chem. 2008; 283(40):27028-27037.

53. Hegde ML, Hegde PM, Holthauzen LM, Hazra TK, Rao KS and Mitra S. Specific Inhibition of NEIL-initiated repair of oxidized base damage in human genome by copper and iron: potential etiological linkage to neurodegenerative diseases. J Biol Chem. 2010; 285(37):28812-28825.

54. Mandal SM, Hegde ML, Chatterjee A, Hegde PM, Szczesny B, Banerjee D, Boldogh I, Gao R, Falkenberg M, Gustafsson CM, Sarkar PS and Hazra TK. Role of human DNA glycosylase Nei-like 2 (NEIL2) and single strand break repair protein polynucleotide kinase 3'-phosphatase in maintenance of mitochondrial genome. J Biol Chem. 2012; 287(4):2819-2829. 